\title{
Paraoxon: An Anticholinesterase That Triggers an Excitotoxic Cascade of Oxidative Stress, Adhesion Responses, and Synaptic Compromise
}

\author{
Karen L.G. Farizatto
}

Ben A. Bahr

Biotechnology Research and Training Center, William C. Friday Laboratory, University of North Carolina - Pembroke, Pembroke, North Carolina, USA.

\section{Doi: 10.19044/esj.2017.c1p4 URL:http://dx.doi.org/10.19044/esj.2017.c1p4}

\begin{abstract}
The anticholinesterase paraoxon (Pxn) is an organophosphate (OP) and the active metabolite of the insecticide parathion. It potently inhibits the enzyme acetylcholinesterase and leads to enhanced glutamate release, diminished GABA uptake, oxidative damage, and neurodegeneration. The resulting increased levels of acetylcholine can trigger seizures and cause neuronal and excitotoxic damage in the brain. The brain susceptibility related to anticholinesterase toxins extends beyond potential brain damage and death from toxic levels of the agent. Asymptomatic low-level exposure to such toxins can also leave the brain vulnerable or even cause it to exhibit neurological problems later in life. The actions of Pxn and similar neurotoxins have been studied in order to examine the events associated with anticholinesterase toxicity in the brain. A recent study demonstrated that Pxn exposure initiates a pathogenic cascade involving seizure events and subsequent signs of damage including unique presynaptic vulnerability and associated behavioral deficits. In addition, Pxn-mediated synaptotoxicity is also associated with enhanced production of oxidative stress as well as integrin adhesion responses. These findings provide a better understanding of the molecular events involved in Pxn toxicity.
\end{abstract}

Keywords: Paraoxon, neurotoxicity, excitotoxicity, anticholinesterase, synapse decline

Paraoxon (Pxn), an anticholinesterase toxin, is in the organophosphate (OP) class of compounds that includes insecticides and military nerve agents (e.g. soman and sarin). Exposure to OP toxins is one of the most common causes of poisoning worldwide, affecting nearly 3 million people each year, of which approximately $15 \%$ die as a result of the poisoning (Eddleston et al., 
2008). Exposure can occur through drinking contaminated water, breathing vapors of the toxins, or subjecting a person's skin to contact with the toxin. In the central nervous system (CNS), such agents classified as neurotoxins and environmental toxins alter cholinergic, glutamatergic, and GABAergic pathways and can lead to seizures, brain damage, and different neurological syndromes.

Understanding the toxic action of anticholinesterase compounds is vital in order to identify pathogenic steps involved and which of these steps lead to disruptions in synaptic integrity and communication. Pxn, the oxidized active metabolite of the insecticide parathion, has become a common research target to study anticholinesterases (Krutak-Krol and Domino, 1985; Harrison et al., 2004; Todorovic et al., 2012; Deshpande et al., 2014). Pxn causes the accumulation of acetylcholine in synapses and results in a cholinergic crisis in the brain (Wei et al. 2014). Enhanced levels of acetylcholine can trigger seizures, long-term behavioral changes, elongated epileptiform action, and reduced cognition (Sánchez-Santed et al., 2004; Millett, 2006; Prager et al., 2015). In humans, acute OP poisoning also leads to respiratory failure and, hence, can cause death (Eddleston et al., 2005).

The brain's susceptibility related to anticholinesterase toxins extends beyond brain damage and death from toxic levels of the agent. Asymptomatic low-level exposure can also leave the brain vulnerable to subsequent brain insults (see Munirathinam and Bahr, 2004). Even neonatal low-doses of exposure to toxic substances such as pesticides can potentiate brain vulnerability to different types of insults in adulthood (Eriksson and Talts, 2000). In addition, repeated exposure to related pesticides has been linked to an increased risk for Alzheimer's disease later in life (Hayden et al., 2010; Sánchez-Santed et al. 2016). Nerve agent exposure is particularly detrimental to the neurodevelopmental processes underlying synaptic connectivity and cognitive ability (Rotenberg and Newmark, 2003). As a result, children are particularly vulnerable to anticholinesterase toxin exposure.

Animal models have been used extensively to understand the effects of anticholinesterase insults and their relationship to brain damage in humans. Harrison and colleagues (2004) found that, in guinea pigs, Pxn caused seizures similar to those caused by the nerve gas soman in humans. Likewise, the anticholinesterase potency of Pxn was similar in macaques and humans (Worek et al., 2011). Furthermore, Rosenberg and colleagues (2017) showed that anticholinesterase-exposed macaques presented severe signs of toxicity (e.g. fasciculations, miosis, salivation, and convulsions), and many died in less than seven hours post-application. This type of research potentially offers an approach to evaluate and better understand the underlying mechanisms of toxins with actions that trigger brain disturbances. 
Certain neurotoxins like Pxn act not only on the cholinergic network, but also affect other neurotransmitter systems involved in excitotoxic propagation. Pxn enhances glutamatergic transmission on hippocampal granule cells. This effect is postulated to occur principally through presynaptic mechanisms (Kozhemyakin et al., 2010). It is noteworthy that the stimulation of nicotinic receptors by itself leads to an increase in glutamate release and thereby increases synaptic transmission in the hippocampus (Sharma and Vijayaraghavan 2003; Alkondon and Albuquerque 2004). Similarly, anticholinesterase agents such as Pxn lead to an over-stimulation of nicotinic and muscarinic receptors through the accumulation of acetylcholine. Pavlovsky and colleagues (2003) previously reported the interaction between these two systems and showed acetylcholine-dependent enhancement of glutamatergic excitatory transmission. Subsequently, Mohammadi and colleagues (2008), also found GABA uptake was significantly reduced in both the cerebral cortex and hippocampus of Pxn-treated rats. Such evidence suggests that acute exposure to anticholinesterase agents not only triggers a cholinergic crisis but occurs in correspondence with the release of excitotoxic levels of glutamate from excitotoxic-sensitive regions of the brain.

Excitotoxic insults are thought to be involved in dendritic and synaptic damage, early toxicological signs that lead to neuronal dysfunction and memory impairment (Munirathinam and Bahr, 2004; Raveh et al., 2002, 2003). Functional imaging during an excitotoxicity study suggests that during seizures, limbic structures such as the amygdala, piriform cortex, entorhinal cortex, and hippocampus are activated (Clifford et al., 1987). Interestingly, hippocampal neurons exhibit an enhanced vulnerability to different types of neuropathogenesis (Mattson, 1990; Bahr et al., 1994), and organophosphate compounds have a significant effect on this brain region (Crino et al., 2002; Harrison et al.,2004), particularly due to the high density of cholinergic and glutamatergic innervations.

Brain damage caused by organophosphate intoxication is not restricted to the primary event induced by the poison, but secondary events associated with the anticholinesterase-induced cellular toxicity were observed across several studies in different cell types and brain regions (see Table 1). In the hippocampus, the anticholinesterase effect was observed in vitro and in vivo by Nallapanemi and colleagues (2006), confirming the ability of Pxn to inhibit the enzyme acetylcholinesterase in this brain region. Pxn exposure comprises different cellular disturbances such as reactive astrocytes, synaptic marker changes, morphological changes, and oxidative stress (Kang et al., 2011; Yu et al., 2012). As found by Jafari and colleagues (2012), Pxn-mediated oxidative damage presented itself not only in brain tissue but also in other organs such as the liver, heart, kidney and spleen. 
Table 1.

Pxn-induced cellular disturbances in different brain regions

Molecular Changes Brain Region / Cells

reduced cholinesterase activity

hippocampus neuroblastoma cell line

enhanced glutamate release hippocampus and amygdala hippocampus and cortex neuroblastoma cell line Reference diminished GABA uptake reduced cell viability oxidative damage human salivary gland cell line

Nallapanemi et al., 2006 Ehrich et al., 1997

Kozhemyakin et al., 2010

Mohammadi et al., 2008

Prins et al., 2010

Prins et al., 2014

In a recent study of Pxn exposure using stable explants of brain tissue, indications of oxidative stress and induced alterations to $\beta 1$-class integrin adhesion molecules were found in correspondence with synaptic compromise (Farizatto et al., 2017). This synaptic compromise was characterized by selectivity of the presynaptic components it affected. Note that, as shown in Table 2, the new study found Pxn-mediated synaptotoxicity associated with oxidative stress as well as integrin adhesion responses both in vitro and in vivo. These molecular indicators of toxicity show consistency with regards Pxnmediated cellular changes across both models. These results further indicate the usefulness of screening studies with brain slices to better understand this type of toxin exposure so that it may be properly treated in such a way that offsets damage caused by the toxic cascade.

Table 2.

Pxn exposure leads to oxidative, adhesive, and synaptic changes in both in vitro and in vivo experiments.

\begin{tabular}{lcc}
\multicolumn{1}{c}{ Toxic Changes } & Pxn-treated hippocampal slice cultures & Pxn-treated rats \\
\hline Oxidative stress & increased 4-HNE adducts & increased 4-HNE adducts \\
Adhesion response & enhanced $\beta 1$ integrin levels & enhanced $\beta 1$ integrin levels \\
Synaptic decline & reduced synapsin IIb and & reduced synapsin IIb levels \\
& synaptophysin levels &
\end{tabular}

OP agents like Pxn may initiate synaptic deterioration in which the presynaptic markers appear to be more susceptible than postsynaptic markers (Kozhemyakin et al., 2010; Farizatto et al., 2017). The synaptic decline profile induced by Pxn can be compared to 1) synaptic disruption reported in excitotoxicity studies using hippocampal slice cultures (Bahr et al., 2002; Karanian et al., 2005; Piwońska et al., 2016) and 2) hippocampal slice synaptic declines from protein accumulation stress studies (Bendiske and Bahr, 2003; Butler et al., 2007; Wisniewski et al., 2011). Synaptic pathology after Pxn exposure has been shown in vitro and in vivo (see Table 2). The illustrative image in Figure 1 shows the synaptic events that are linked to inhibition of acetylcholinesterase. Pxn exposure leads to excess of acetylcholine activity and enhanced glutamate release, and one or both of these changes can lead to seizure, excitotoxicity and brain damage. These findings suggest that Pxn affects the native composition of important CNS synapses and their control of important brain functions. 


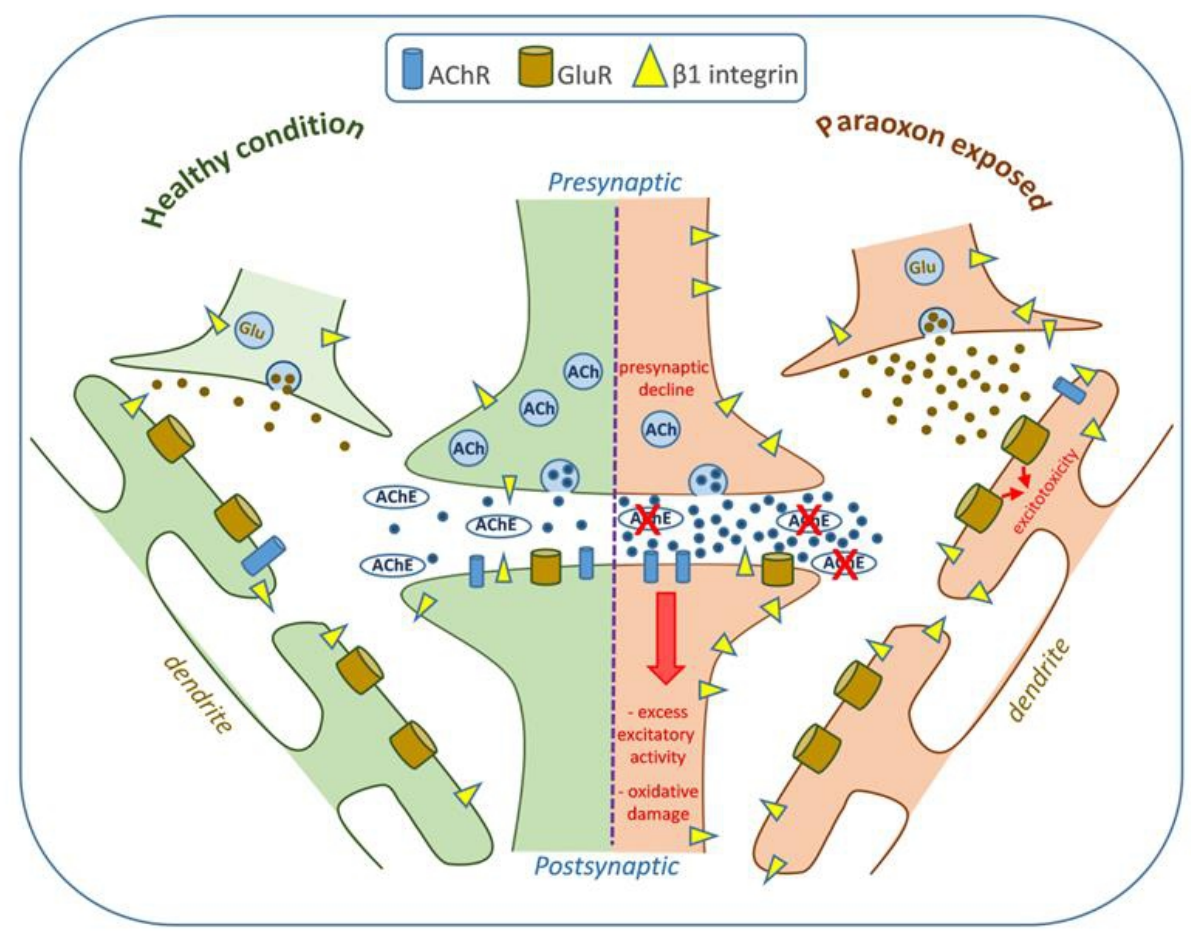

Figure 1: Schematic Illustration of paraoxon-mediated cellular disturbances on brain tissue.

The anticholinesterase paraoxon leads to over-excitatory activity through excess of acetylcholine and enhanced glutamate release in the synaptic cleft, which elicits a cholinergic crisis and excitotoxic damage. Note, the potent inhibition of the enzyme acetylcholinesterase (AChE) by paraoxon exposure also causes presynaptic compromise, adhesion response and oxidative damage.

The degradation of the neurotransmitter acetylcholine by acetylcholinesterase is critical for the maintenance and homeostasis of synaptic integrity and communication. Acute and chronic exposure to compounds like Pxn can lead to neurotoxic effects associated with the human conditions called cholinergic syndrome, intermediate syndrome, organophosphate-induced delayed polyneuropathy, and chronic organophosphate-induced neuropsychiatric disorder. It is very important to understand the actions of related neurotoxins and environmental toxins on synaptic mechanisms and CNS pathways, including cholinergic, glutamatergic, GABAergic, as well as antioxidant systems. The brain's susceptibility to anticholinesterase toxins and the distinct effects on synapses can leave exposed individuals vulnerable to symptoms and neurological problems for the rest of their lives. 


\section{Acknowledgements}

This material is based upon work supported by U.S. Army Research Office and the Department of Defense Research and Education Program under grant number W911NF-15-1-0432. The research was also supported by National Institutes of Health grant 5R25GM077634-04. The funding agencies had no role in data collection or decision to publish. We thank Catherine M. Parisian for excellent proofreading and editing this article.

\section{References:}

1. Alkondon M, Albuquerque EX (2004) The nicotinic acetylcholine receptor subtypes and their function in the hippocampus and cerebral cortex. Progress in Brain Research 145:109-120.

2. Bahr BA, Abai B, Gall C, Vanderklish PW, Hoffman KB, Lynch G (1994) Induction of $\beta$-amyloid-containing polypeptides in hippocampus: Evidence for a concomitant loss of synaptic proteins and interactions with an excitotoxin. Experimental Neurology 129:81-94.

3. Bahr BA, Bendiske J, Brown QB, Munirathinam S, Caba E, Rudin M, Urwyler S, Sauter A, Rogers G (2002) Survival signaling and selective neuroprotection through glutamatergic transmission. Experimental Neurology 174:137-147.

4. Bendiske J, Bahr BA (2003) Lysosomal activation is a compensatory response against protein accumulation and associated synaptopathogenesis--an approach for slowing Alzheimer disease? Journal of Neuropathology and Experimental Neurology 62:451-463.

5. Butler D, Bendiske J, Michaelis ML, Karanian DA, Bahr BA (2007) Microtubule-stabilizing agent prevents protein accumulation-induced loss of synaptic markers. European Journal of Pharmacology 562:2027.

6. Clifford DB, Olney JW, Maniotis A, Collins RC, Zorumski CF (1987) The functional anatomy and pathology of lithium-pilocarpine and high-dose pilocarpine seizures. Neuroscience 23: 953-968.

7. Crino PB, Jin H, Shumate MD, Robinson MB, Coulter DA, BrooksKayal AR (2002) Increased expression of the neuronal glutamate transporter (EAAT3/EAAC1) in hippocampal and neocortical epilepsy. Epilepsia 43:211-218.

8. Deshpande LS, Phillips K, Huang B, Delorenzo RJ (2014) Chronic behavioral and cognitive deficits in a rat survival model of paraoxon toxicity. Neurotoxicology 44:352-357.

9. Eddleston M, Buckley NA, Eyer P, Dawson AH (2008) Management of acute organophosphorus pesticide poisoning. The Lancet 371: 597607. 
10. Eddleston M, Gunnell D, Karunaratne A, de Silva D, Sheriff MH, Buckley NA (2005) Epidemiology of intentional self-poisoning in rural Sri Lanka. The British Journal of Psychiatry 187:583-584.

11. Ehrich M, Correll L, Veronesi B (1997) Acetylcholinesterase and neuropathy target esterase inhibitions in neuroblastoma cells to distinguish organophosphorus compounds causing acute and delayed neurotoxicity. Fundamental and Applied Toxicology 38:55-63.

12. Eriksson P, Talts U (2000) Neonatal exposure to neurotoxic pesticides increases adult susceptibility: a review of current findings. Neurotoxicology 21:37-47.

13. Farizatto KLG, McEwan SA, Naidoo V, Nikas SP, Shukla VG, Almeida MF, Byrd A, Romine HW, Karanian DA, Makriyannis A, Bahr BA (2017) Inhibitor of endocannabinoid deactivation protects against in vitro and in vivo neurotoxic effects of paraoxon. Journal of Molecular Neuroscience. doi: 10.1007/s12031-017-0963-4. Epub ahead of print.

14. Harrison PK, Sheridan RD, Green AC, Scott IR, Tattersall JE (2004) A guinea pig hippocampal slice model of organophosphate-induced seizure activity. Journal of Pharmacology and Experimental Therapeutics 310:678-686.

15. Hayden KM, Norton MC, Darcey D, Ostbye T, Zandi PP, Breitner JC, Welsh-Bohmer KA; Cache County Study Investigators (2010) Occupational exposure to pesticides increases the risk of incident AD: the Cache County study. Neurology 74:1524-1530.

16. Jafari M, Salehi M, Asgari A, Ahmadi S, Abbasnezhad M, Hajihoosani R, Hajigholamali M (2012) Effects of paraoxon on serum biochemical parameters and oxidative stress induction in various tissues of Wistar and Norway rats._Environmental Toxicology and Pharmacology 34:876-887.

17. Kang WH, Simon MJ, Gao S, Banta S, and Morrison B $3^{\text {rd }}$ (2011) Attenuation of astrocyte activation by TAT-mediated delivery of a peptide JNK inhibitor. Journal of Neurotrauma 28:1219-1228.

18. Karanian DA, Brown QB, Makriyannis A, Bahr BA (2005) Blocking cannabinoid activation of FAK and ERK1/2 compromises synaptic integrity in hippocampus. European Journal of Pharmacology 508:4756.

19. Kozhemyakin M, Rajasekaran K, Kapur J (2010) Central cholinesterase inhibition enhances glutamatergic synaptic transmission. Journal of Neurophysiology 103:1748-1757.

20. Krutak-Krol H, Domino EF (1985) Comparative effects of diazepam and midazolam on paraoxon toxicity in rats. Toxicology and Applied Pharmacology 81:545-550. 
21. Mattson RH (1990) Selection of drugs for the treatment of epilepsy. Seminars in Neurology 10:406-413.

22. Millett PD (2006) The biological and toxin weapons convention. Revue Scientifique et Technique - International Office of Epizootics 25:35-52.

23. Mohammadi M, Ghani E, Ghasemi A, Khoshbaten A, Asgari A (2008) Synaptosomal GABA uptake decreases in paraoxon-treated rat brain. Toxicology 244: 42-48.

24. Munirathinam S, Bahr BA (2004) Repeated contact with subtoxic soman leads to synaptic vulnerability in hippocampus. Journal of Neuroscience Research 77:739-746.

25. Nallapanemi A, Liu J, Karanth S, Pope C (2006) Modulation of paraoxon toxicity by the cannabinoid receptor agonist WIN 55,212-2. Toxicology 227:173-183.

26. Pavlovsky L, Browne RO, Friedman M (2003) Pyridostigmine enhances glutamatergic transmission in hippocampal CA1 neurons. Experimental Neurology 179:181-187.

27. Piwońska M, Szewczyk A, Schröder UH, Reymann KG, Bednarczyk I (2016) Effectors of large-conductance calcium-activated potassium channel modulate glutamate excitotoxicity in organotypic hippocampal slice cultures. Acta Neurobiologiae Experimentalis 76: 20-31.

28. Prager EM, Figueiredo TH, Long RP $2^{\text {nd }}$, Aroniadou-Anderjaska V, Apland JP, Braga MF (2015) LY293558 prevents soman-induced pathophysiological alterations in the basolateral amygdala and the development of anxiety. Neuropharmacology 89:11-18.

29. Prins JM, Chao CK, Jacobson SM, Thompson CM, George KM (2014) Oxidative stress resulting from exposure of a human salivary gland cells to paraoxon: an in vitro model for organophosphate oral exposure._Toxicology in Vitro 28:715-721.

30. Prins JM, George KM, Thompson CM (2010) Paraoxon-induced protein expression changes to SH-SY5Y cells. Chemical Research in Toxicology 23:1656-1662.

31. Raveh L, Brandeis R, Gilat E, Cohen G, Alkalay D, Rabinovitz I, Sonego H, Weissman BA (2003) Anticholinergic and antiglutamatergic agents protect against soman-induced brain damage and cognitive dysfunction. Toxicological Sciences 75:108-116.

32. Raveh L, Weissman BA, Cohen G, Alkalay D, Rabinovitz I, Sonego H, Brandeis R (2002) Caramiphen and scopolamine prevent somaninduced brain damage and cognitive dysfunction. Neurotoxicology 23:7-17. 
33. Rosenberg YJ, Mao L, Jiang X, Lees J, Zhang L, Radic Z, Taylor $\mathrm{P}$ (2017) Post-exposure treatment with the oxime RS194B rapidly reverses early and advanced symptoms in macaques exposed to sarin vapor. Chemico-Biological Interactions 274:50-57.

34. Rotenberg JS, Newmark J (2003) Nerve agent attacks on children: diagnosis and management. Pediatrics 112:648-658.

35. Sánchez-Santed F, Cañadas F, Flores P, López-Grancha M, Cadona D (2004) Long-term functional neurotoxicity of paraoxon and chlorpyrifos: beha vioural and pharmacological evidence. Neurotoxicology and Teratology 26:305-317.

36. Sánchez-Santed F, Colomina MT, Herrero Hernández E (2016) Organophosphate pesticide exposure and neurodegeneration. Cortex 74:417-426.

37. Sharma G, Vijayaraghavan S (2003) Modulation of presynaptic store calcium induces release of glutamate and postsynaptic firing. Neuron 38:929-939.

38. Todorovic MS, Cowan ML, Balint CA, Sun C, Kapur J (2012) Characterization of status epilepticus induced by two organophosphates in rats. Epilepsy Research 101:268-276.

39. Wei Z, Liu YQ, Zhou XB, Luo Y, Huang CQ, Wang YA, Zheng $\mathrm{ZB}$, Li S (2014) New efficient imidazolium aldoxime reactivators for nerve agent-inhibited acetylcholinesterase. Bioorganic and Medicinal Chemistry Letters 24:5743-5748.

40. Wisniewski ML, Hwang J, Bahr BA (2011) Submicromolar Aß42 reduces hippocampal glutamate receptors and presynaptic markers in an aggregation-dependent manner. Biochimica et Biophysica Acta 1812:1664-1674.

41. Worek F, Aurbek N, Wille T, Eyer P, Thiermann H (2011) Kinetic analysis of interactions of paraoxon and oximes with human, Rhesus monkey, swine, rabbit, rat and Guinea pig acetylcholinesterase. Toxicology Letters 200:19-23

42. Yu F, Wang Z, Tchantchou F, Chiu CT, Zhang Y, Chuang DM (2012) Lithium ameliorates neurodegeneration, suppresses neuroinflamation, and improves behavioral perfomance in a mouse model of traumatic brain injury. Journal of Neurotrauma 29:362-374. 